\title{
Comparison of two oral probiotic preparations in a randomized crossover trial highlights a potentially beneficial effect of Lactobacillus paracasei NCC2461 in patients with allergic rhinitis
}

Yannick Perrin ${ }^{1 \dagger}$, Sophie Nutten ${ }^{2 * \dagger}$, Régine Audran ${ }^{1}$, Bernard Berger ${ }^{2}$, Rodrigo Bibiloni ${ }^{2,3}$, Jacqueline Wassenberg ${ }^{1}$, Nathalie Barbier ${ }^{1}$, Vincent Aubert ${ }^{1}$, Julie Moulin², Anurag Singh² ${ }^{2}$ Corinne Magliola ${ }^{2}$, Annick Mercenier ${ }^{2}$ and François Spertini ${ }^{1}$

\begin{abstract}
Background: There is promising but conflicting evidence to recommend the addition of probiotics to foods for prevention and treatment of allergy. Based on previous studies with fermented milk containing Lactobacillus paracasei NCC2461, we aimed to compare the effect of a powder form of the latter probiotic with the effect of a blend of Lactobacillus acidophilus ATCC SD5221 and Bifidobacterium lactis ATCC SD5219 in patients with allergic rhinitis.

Methods: A double-blind, randomized, cross-over study, involving 31 adults with allergic rhinitis to grass pollen, was performed outside the grass pollen season (registration number: NCT01233154). Subjects received each product for 4-weeks in two phases separated by a wash-out period of 6 to 8 weeks. A nasal provocation test was performed before and after each 4-week product intake period, and outcome parameters (objective and subjective clinical symptoms; immune parameters) were measured during and/or 24 hours after the test.

Results: Out of the 31 subject enrolled, 28 completed the study. While no effect was observed on nasal congestion (primary outcome), treatment with NCC2461 significantly decreased nasal pruritus (determined by VAS), and leukocytes in nasal fluid samples, enhanced IL-5, IL-13 and IL-10 production by peripheral blood mononuclear cells in an allergen specific manner and tended to decrease IL-5 secretion in nasal fluid, in contrast to treatment with the blend of L. acidophilus and B. lactis.
\end{abstract}

Conclusions: Despite short-term consumption, NCC2461 was able to reduce subjective nasal pruritus while not affecting nasal congestion in adults suffering from grass pollen allergic rhinitis. The associated decrease in nasal fluid leukocytes and IL-5 secretion, and the enhanced IL-10 secretion in an allergen specific manner may partly explain the decrease in nasal pruritus. However, somewhat unexpected systemic immune changes were also noted. These data support the study of NCC2461 consumption in a seasonal clinical trial to further demonstrate its potentially beneficial effect.

Keywords: Allergic rhinitis, Immunomodulation, Nasal provocation test, Lactobacillus paracasei, Clinical trial, Grass pollen

\footnotetext{
* Correspondence: sophie.nutten@rdls.nestle.com

${ }^{\dagger}$ Equal contributors

${ }^{2}$ Nestlé Research Centre, Lausanne, Switzerland

Full list of author information is available at the end of the article
} 


\section{Introduction}

Current available treatments for allergic rhinitis are not devoid of side-effects and primary prevention strategies are non-existent. This has led to the search for new approaches to prevent or reduce allergic symptoms and improve quality of life of patients. In this context, and regarding its safety profile, interest in nutritional intervention for allergy management has been growing. Recent scientific evidence from pre-clinical studies and human clinical trials has highlighted different nutritional interventions such as vitamins, lipids, dietary polyphenols and probiotics as promising agents that can impact both allergic sensitization and alleviate allergic symptoms [1-3]. Probiotics are defined as "live microorganisms which when administered in adequate amounts confer a health benefit on the host" [4]. The "hygiene hypothesis" suggested that modern living conditions may have affected the initial establishment of the intestinal microbiota, with subsequent impact on the development of the GALT (gut-associated lymphoid tissue) at an early age, and therefore on the regulation of local and systemic immune responses [5]. In addition, several studies pointed out differences in the gut microbial composition between infants developing allergy or not, suggesting a crucial role of the intestinal microbiota on the immune system orientation [6-9].

Most clinical studies in the field of probiotics and allergy have focused on the prevention or treatment of atopic dermatitis, with divergent outcomes likely resulting from different probiotic strains, target populations, and study designs [10-15]. Although evidence of a beneficial effect of probiotics on allergic respiratory symptoms is still conflicting [16,17], a few studies conducted in children and adults with allergic rhinitis suggested a beneficial effect of the consumption of specific probiotic strains belonging to the Lactobacillus casei, Bifidobacterium longum, Bifidobacterium lactis or Lactobacillus paracasei species [18-22]. We also recently showed in a crossover, placebo controlled clinical trial that the administration of a fermented milk containing Lactobacillus paracasei NCC2461 yielded encouraging results on allergic rhinitis symptoms and immune biomarkers [23]. To reinforce our previous observations on Lactobacillus paracasei NCC2461, we conducted the present study to compare the effect of a 4-week consumption of two probiotic preparations on clinical and biological responses to a well-reproducible nasal provocation test (NPT) with grass pollen, in adult volunteers with grass pollen allergic rhinitis. The second probiotic preparation (combination of $L$. acidophilus SD5221 and B. lactis SD5219) was chosen based on promising results obtained in children with birch pollen allergy [24].

\section{Material and methods Population}

Thirty-one adult volunteers with a history of grass pollen allergic rhinitis were enrolled (Figure 1) in a single center study (CHUV, Lausanne, Switzerland) on the basis of the following inclusion criteria: (1) age between 18 and 35 years; (2) a history of allergic rhinitis during the latest grass pollen season confirmed by positive skin prick test (SPT) to grass pollen (wheal diameter $>3 \mathrm{~mm}$ ); and (3) a positive response to a NPT with grass pollen (combined nasal reaction threshold $\leq 10^{\prime} 000$ standardized quality units (SQs)/ml grass pollen or less at the screening/ inclusion phase). Volunteers were excluded from the study when presenting any medical condition that could influence the study (pregnancy, viral or bacterial airway infection, active allergic rhinitis), or uncontrolled asthma (peak expiratory flow $<20 \%$ of volunteer's best personal value), when treated with antihistamine or antibiotics less than two weeks before enrolment or during the trial. Furthermore they were also excluded when presenting with allergic rhinitis to tree pollen or perennial allergic rhinitis. Clinical research protocol was approved by the Ethical Review Board of the Faculty of Biology and Medicine, Lausanne ( $\left.\mathrm{N}^{\circ} 153 / 07\right)$ as well as by Swiss Regulatory Authorities (Swissmedic, Bern) and all enrolled subjects provided informed consent before the start of the study.

\section{Study products}

Powder sachets contained maltodextrin and probiotics $\left(\approx 10^{10}\right.$ colony forming unit (cfu) each), either $L$. paracasei NCC2461 (CNCM I-2116; ST11; Nestlé, Switzerland) or a blend (1:3) of L. acidophilus ATCC SD5221 and B. lactis ATCC SD5219 (Danisco Cultures, USA). They were kept at $4^{\circ} \mathrm{C}$ until consumption. The 31 subjects were randomized according to a computer generated allocation schedule to receive one of the individually coloured test products and instructed to dilute once a day the content of one sachet into $2 \mathrm{dl}$ of cold milk just before consumption on an empty stomach. Quality control of the powder sachets was carried out for product release, in the middle, and at the end of the study, to ensure viability of the tested probiotics.

\section{Study protocol}

The study was designed as a randomized, double-blind, two-treatment, cross-over clinical trial, performed outside of the grass pollen season (between August 2007 and March 2008; registration number: NCT01233154) to evaluate the effect of a 4-week consumption of two probiotic formulations (period A and period C) on clinical and biological responses to NPT (Figure 2). Both investigators and subjects were aware that the aim of the study was to test two different probiotic preparations, but 


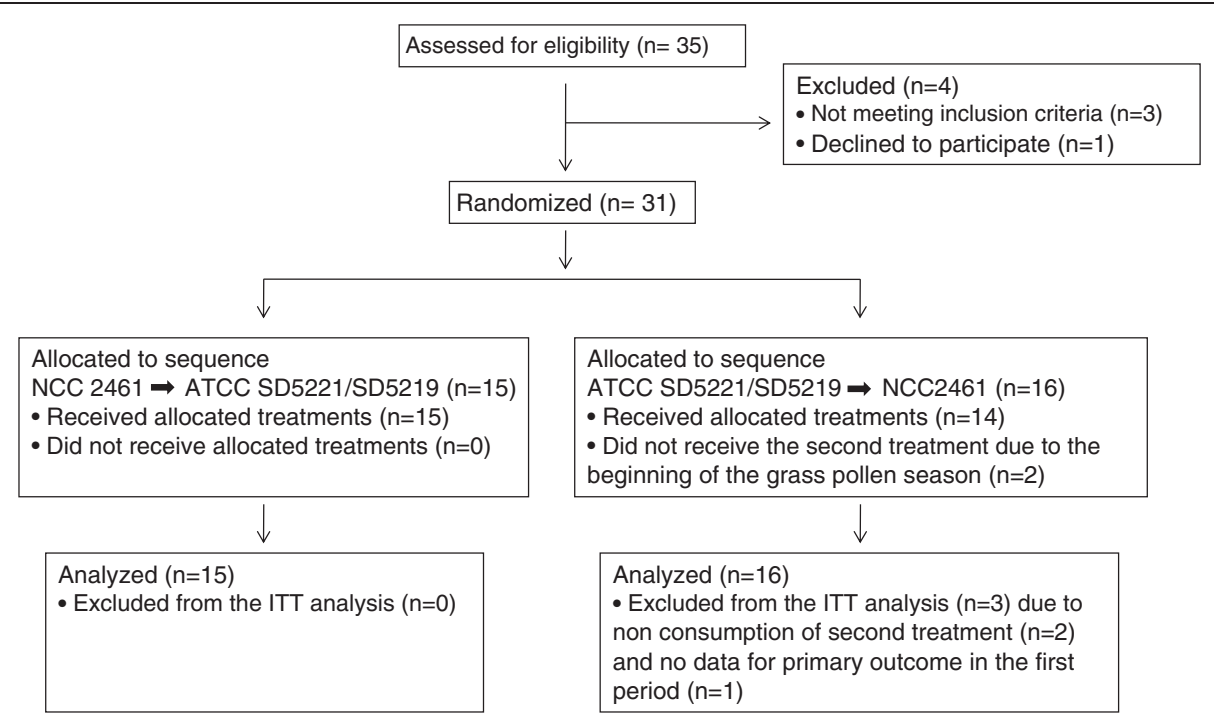

Figure 1 Participants' recruitment and inclusion flow chart.

they were both blinded with regards to the probiotic preparation. The choice of the duration (four weeks) of the treatment period was mainly based on the encouraging results obtained with $L$. paracasei NCC2461 in a previous clinical trial [23]. Products were supplied before each period of product consumption. Both treatment periods were separated by a wash-out period (period B) of six to eight weeks. A NPT was performed before and after each treatment period, and outcome parameters were measured during and/or 24 hours after each NPT. Non solicited adverse events were recorded all along the trial. Compliance was evaluated on subjects' product consumption reported in diary cards and from stool samples analysis of studied probiotics at the beginning and the end of each treatment period.

The primary outcome of the study was defined as the difference before and after treatment with each probiotic preparation on subjective nasal congestion by visual analogical scale (VAS). We additionally compared the two treatments in their capacity to modify this endpoint. Secondary endpoints included 1) differences before and after treatment in allergen dose levels necessary to reach the combined reaction threshold. Each clinical criterion and their respective thresholds were also examined separately as secondary endpoints and compared between the two treatment arms; 2) nasal pruritus evaluated by VAS as well as immune markers from blood and nasal samples as described below.

\section{NPT and clinical assessment}

The NPT was performed as described previously [25-27], before and after each 4-week product intake period. The "combined nasal reaction threshold" was defined as the dose level of the allergen extract for which at least two of the three following clinical criteria were fulfilled: (1) $\geq 5$ sneezes in first ten minutes after challenge; (2) $\geq 0.5$ g nasal secretion in the first ten minutes after challenge (above diluent value); (3) a decrease in peak nasal inspiratory flow $(\mathrm{PNIF}) \geq 40 \%$ or minimal cross-sectional area (MCA) $\geq 30 \%$ of baseline (diluent value), whichever came first [28].

\section{Nasal samples and immune markers}

Nasal samples were collected before NPT, 10 min. after the $1^{\prime} 000 \mathrm{SQ} / \mathrm{ml}$ challenge and $24 \mathrm{~h}$ after NPT: cells

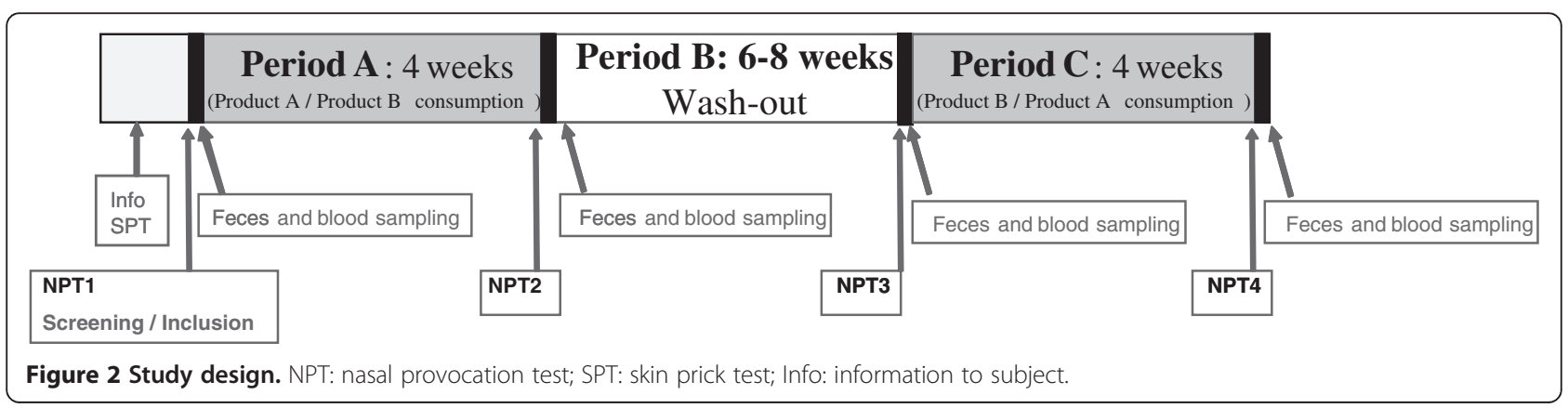


were obtained by scraping the inferior turbinate and nasal septum of a single nostril three times with an endocervical sampling brush (cyto-brosse, Charles Berdat, Switzerland) as previously described [19], and fluids by application of 2 filter paper strips $\left(6 \times 30 \mathrm{~mm}\right.$, Whatman $\mathrm{N}^{\circ} 903$, Whatman Paper Ltd., Maidstone, UK) in the controlateral nostril (inferior turbinate and nasal septum) for $10 \mathrm{~min}$ [29]. After removal, the strips were placed in a $2 \mathrm{ml}$ silicone-coated tube in dry-ice and stored at $-80^{\circ} \mathrm{C}$ until processed. Papers were eluted in $1 \mathrm{ml}$ of Tris buffer $0.1 \mathrm{M}, \mathrm{pH}$ 7.4, $0.002 \%$ Tween 20 by strong agitation at $4{ }^{\circ} \mathrm{C}$ during at least $2 \mathrm{~h}$ [30]. Cytokines (IL-5 and TNF $\alpha$ ) were measured in nasal fluid by multiplexed bead-based flow cytometry (Fluorokine multiplex kit, R\&D Systems, Inc, Minneapolis, USA) using a Luminex 100 analyser (detection limits: 0.21 and $0.59 \mathrm{pg} / \mathrm{ml}$ respectively) [23].

\section{Blood samples and immune markers}

Serum samples $(10 \mathrm{ml})$ for measuring specific IgE and IgG4 levels to grass pollen, were collected $24 \mathrm{~h}$ after NPT, before and after each product consumption period (Figure 2) and stored at $-80^{\circ} \mathrm{C}$ until analysis. Specific IgG4 and IgE were measured by the CAP System (UniCAP 100, Pharmacia Diagnostics, Uppsala, Sweden). For the in vitro stimulation of peripheral blood mononuclear cells (PBMCs), whole blood was collected 24 h after NPT (before and after each product consumption period), in sodium citrate tubes (Vacutainer ${ }^{\circ} \mathrm{CPT}^{\mathrm{m}}$, BD, Basel, Switzerland), and PBMCs purified by centrifugation over a density gradient. After washing, PBMCs were stored in liquid nitrogen until use. PBMCs were thawed, and cultured at $2 \times 10^{6} \mathrm{cell} / \mathrm{ml}$ as previously described [23] in the presence of $90 \mu \mathrm{g} / \mathrm{ml}$ of grass pollen extract (ALK Wässrig SQ; ALK-Abello, Hørsholm, DK), or a cocktail of vaccine antigens $(5 \mu \mathrm{l} / \mathrm{ml}$ Candida mannan (NIBSC, London, UK), $10 \mu \mathrm{g} / \mathrm{ml}$ tetanus toxoid, $5 \mu \mathrm{g} / \mathrm{ml}$ Tuberculin Purified Protein derivative (PPD, SSI, Copenhagen, DK)), or medium alone. The supernatants were collected after 6 days of culture, and stored at $-20^{\circ} \mathrm{C}$ until evaluation of their cytokine contents (IL-5, IL-8, IL-10 and IFN $\gamma$ by multiplex flow cytometry as described above and IL-13 by ELISA (Diaclone, Besançon, F); detection limits: 0.21, 0.50, 0.34, 1.28 and $0.8 \mathrm{pg} / \mathrm{ml}$ respectively).

\section{Fecal samples}

Fecal samples were collected as described in Figure 2 into sterile tubes maintained in anaerobic conditions using the AnaeroGen system (Oxoid, UK) and kept at $<10^{\circ} \mathrm{C}$ until processing within 12 hours. Briefly, $100 \mathrm{mg}$ of faeces sample were resuspended in sterile saline and serial dilutions performed $\left(10^{-2}-10^{-6}\right)$. Then, $100 \mu \mathrm{L}$ of each dilution were plated on MRS and BSM media [31]. Plates were incubated at $37^{\circ} \mathrm{C}$ in anaerobic conditions. After $48 \mathrm{~h}$, the whole bacteria lawn was scraped off, resuspended in $10 \mu \mathrm{L}$ of $\mathrm{NaCl} 0.9 \%$ and centrifuged for $3 \mathrm{~min}$ at $5000 \mathrm{rpm}$. The pellet was dissolved in $1 \mathrm{~mL} \mathrm{NaCl} \mathrm{0.9 \%}$ and the DNA was extracted by mechanical disruption (bead-beater) to perform PCR using strain (L. paracasei NCC2461 and L. acidophilus ATCC SD5221) or cluster of strains (B. lactis ATCC SD5219) specific primers. Results were expressed as presence or absence of the bacterial strains in the samples.

\section{Statistical analysis}

Demographic and baseline characteristics were documented by descriptive statistics. According to a previous study, knowing that the within subject SD on the primary outcome was 0.6 , a sample size of 27 subjects allows to predict a difference of 0.3 (VAS scale) with a power of $80 \%$ and a type I error of 0.05 [23]. Taking into account a drop-out rate of $15 \%, 32$ subjects were planned to be enrolled. Comparison of primary outcome parameters before and after each probiotic treatment was analyzed with Wilcoxon Signed-Rank Test for each of the products tested. For each treatment, the comparisons of scores between post- and pre-treatment phases were analyzed by linear mixed model with phase, allergen concentration and period as fixed effects and subjects as random effect. For each treatment, the comparisons of the number of subjects for reaction thresholds between post- and pretreatment phases were estimated by Cochran-MantelHaenszel statistics based on table thresholds with the hypothesis that mean thresholds differ. Immunological data were analyzed by Wilcoxon Signed-Rank Test or Paired t-Test according to normality of data. The same analyses were performed on the "post-treatment minus pre-treatment" differences for all outcome parameters, when comparing the two probiotic preparations. All statistical analyses were done with SAS software (version 9.1).

\section{Results}

\section{Demographic and baseline data}

Thirty-one patients (21 males and 10 females) aged 18 to 35 years were enrolled into the study (Table 1; Figure 1). All the results concerning these subjects constituted the intention-to-treat (ITT) data set. Twenty-three percent of enrolled subjects were smokers, with an average of $6 \pm 3$ cigarettes per day. Later enrolment of two subjects led to their exclusion from participating in the second treatment period, because of the overlap of the second period of

Table 1 Demographic data at enrolment

\begin{tabular}{lllll}
\hline & $\mathbf{n}$ & Mean SD & Min & Max \\
\hline Age (years) & 31 & 26.83 .7 & 20.2 & 34.6 \\
Height $(\mathrm{cm})$ & 31 & 173.87 .3 & 161.0 & 190.0 \\
Weight $(\mathrm{kg})$ & 31 & 69.712 .5 & 49.0 & 98.0 \\
Body mass index $\left(\mathrm{BMl}, \mathrm{kg} / \mathrm{m}^{2}\right)$ & 31 & 22.973 .25 & 17.36 & 30.93 \\
\hline
\end{tabular}


treatment with the beginning of the pollen season. For one subject, the primary outcome parameter was not evaluated in the first treatment period. Therefore, twenty-eight subjects completed the study.

\section{Compliance and safety}

Compliance with product consumption was good all over the study. According to diary cards, the mean duration of consumption of L. paracasei NCC2461 and blend of L. acidophilus ATCC SD5221 and B. lactis ATCC SD5219 were $28.4 \pm 0.4$ and $31.1 \pm 0.4$ days, respectively. Consumption of both probiotic preparations was confirmed by their respective presence in the subject's feces after period $A$ and $C$ (Table 2). Importantly, the wash-out period duration was considered to be well-adapted considering the absence of strain detection at the end of the wash-out period ("Before period C" column) in all except 2 volunteers. For patient 29, the detection of $L$. paracasei NCC2461 is probably a false positive result, since this strain was not detected before the wash-out period. For patient 32, we suspect that other Bifidobacterium spp strain(s), closely related to B. lactis ATCC SD5219, could have been detected because of the use of species and not strain related primers. There was no noticeable clinical issue and no formulation-related adverse event during the study.

\section{Clinical outcomes}

With regards to the primary outcome, there was no significant difference at all grass pollen concentrations between the post-treatment and the pre-treatment scores for both probiotic products on nasal congestion scores (as determined by VAS, Figure 3). Furthermore, neither of the two probiotic treatments significantly affected the objective clinical parameters (PNIF, MCA, nasal secretions, sneezes, and the combined nasal reaction threshold). However, subjective nasal pruritus (as determined by VAS), one of the main secondary outcomes, was globally significantly lower after L. paracasei NCC2461 treatment than before (ITT analysis; $\mathrm{p}=0.005$ ) (Figure 4A) in contrast to the blend of L. acidophilus ATCC SD5221 and B. lactis ATCC SD5219 which had no effect on nasal pruritus scores (Figure 4B). Moreover, when the difference between post-treatment and pre-treatment pruritus scores was compared between both products, a significantly greater reduction in pruritus score was shown with $L$. paracasei NCC2461 treatment, as compared to the L. acidophilus and B. lactis blend (ITT analysis: $\mathrm{p}=0.005$ ) (Figure 4C).

\section{Nasal cell counts and immune markers}

Interestingly, after treatment with $L$. paracasei NCC2461, the percentage of total leukocytes 24 hours after NPT was significantly lower than in the pre-treatment phase
( $\mathrm{p}=0.009$; ITT analysis) (Figure $5 \mathrm{~A})$. However, the posttreatment minus pre-treatment difference in leukocyte percentage did not significantly differ between treatments. No significant effect on relative nasal cell counts resulted from treatment with the blend of $L$. acidophilus ATCC SD5221 and B. lactis ATCC SD5219 (Figure 5B).

In the $L$. paracase $i$ treatment arm, the post-treatment nasal fluid TNF $\alpha$ contents were not significantly different from the pre-treatment ones $(0.028 \pm 0.106 \mathrm{pg} / \mathrm{ml}, p=0.55)$, a trend was observed toward a decrease in IL-5 measured 24 hours after NPT $(-0.756 \pm 1.412, p=0.08$; ITT analysis $)$. No effect of treatment with the blend of $L$. acidophilus and $B$. lactis was observed on nasal fluid cytokine levels of IL-5 and TNF- $\alpha$ (data not shown). The comparison of the post- minus pre-treatment difference in nasal fluid cytokine contents between the two treatments did not show significant differences for IL- 5 or TNF- $\alpha$.

\section{Cell mediated immunity markers}

None of the probiotic treatment induced any change in the spontaneous secretion by PBMC (Table 3; NS). This suggests that both probiotic treatments are safe and do not affect the immune system steady state. Treatment with L. paracasei NCC2461 was associated with a significant increase in the secretion of IL-5, IL-8, IL-10 and IL-13 by grass pollen-stimulated PBMCs, while no effect on IFN- $\gamma$ secretion was observed (Table 3). In contrast, treatment with the blend of L. acidophilus ATCC SD5221 and B. lactis ATCC SD5219 did not result in differences in cytokine secretion by grass pollen-stimulated PBMCs (Table 3). Comparison of cytokine changes between both treatments did not show significant differences for IL-5, IL-10, IL-13, and IFN- $\gamma$ secretion by grass pollenstimulated PBMCs, whereas IL-8 secretion was significantly enhanced with $L$. paracasei NCC2461 treatment, as compared to the other treatment $(\mathrm{p}=0.03$; ITT) (data not shown).

Treatment with L. paracasei NCC2461 was also associated with a significant increase in IL-10 secretion by PBMCs stimulated by a mix of recall vaccine antigens $(\mathrm{p}=0.01 ;$ ITT) (Table 3$)$, whereas the other treatment did not have such an effect. A significant difference between both treatments was found for IL-10 secretion with higher levels obtained after L. paracasei NCC2461 treatment ( $\mathrm{p}=0.008$; ITT) (data not shown).

\section{Serological immune markers}

Serum anti-grass pollen specific IgE were significantly increased after both treatments $(56.9 \pm 11.6 \mathrm{KU} / \mathrm{L}$ and $64.6 \pm 12.4 \mathrm{KU} / \mathrm{L}$, mean \pm SEM) before and after $L$. paracasei NCC2461 treatment, respectively $(\mathrm{p}=0.02)$ and $63.6 \pm 12.6 \mathrm{KU} / \mathrm{L}$ and $66.3 \pm 12.4 \mathrm{KU} / \mathrm{L}$ before and after $L$. acidophilus ATCC SD5221 and B. lactis ATCC SD5219 treatment, respectively $(\mathrm{p}=0.04)$. No change in specific 
Table 2 Detection of $L$. paracasei NCC2461 and B. lactis SD5219 strains during clinical trial phases (before and after periods $A$ and $C$ )

\begin{tabular}{|c|c|c|c|c|c|c|c|c|}
\hline \multirow[b]{2}{*}{ Subject study number } & \multicolumn{2}{|c|}{ Before period $\mathrm{A}$} & \multicolumn{2}{|c|}{ After period $\mathrm{A}$} & \multicolumn{2}{|c|}{ Before period C } & \multicolumn{2}{|c|}{ After period C } \\
\hline & A & B & A & B & A & B & A & B \\
\hline 1 & & $\checkmark$ & $\checkmark$ & & & & & $\checkmark$ \\
\hline 3 & & & $\checkmark$ & & & & & $\checkmark$ \\
\hline 4 & & & $\checkmark$ & $\checkmark$ & & & & $\checkmark$ \\
\hline 5 & & & & $\checkmark$ & & & & \\
\hline 6 & & & & $\checkmark$ & & & $\checkmark$ & \\
\hline 7 & & & $\checkmark$ & & & & & $\checkmark$ \\
\hline 8 & & & & $\checkmark$ & & & $\checkmark$ & \\
\hline 9 & & & & $\checkmark$ & & & $\checkmark$ & $\checkmark$ \\
\hline 10 & & & & $\checkmark$ & & & $\checkmark$ & \\
\hline 11 & & $\checkmark$ & & & & & & $\checkmark$ \\
\hline 12 & & & $\checkmark$ & & & & $\checkmark$ & $\checkmark$ \\
\hline 13 & & & & & & & & $\checkmark$ \\
\hline 14 & & & & & & & & $\checkmark$ \\
\hline 15 & & & $\checkmark$ & & & & & $\checkmark$ \\
\hline 16 & & & & $\checkmark$ & & & $\checkmark$ & \\
\hline 17 & & & & $\checkmark$ & & & $\checkmark$ & \\
\hline 18 & & & & $\checkmark$ & & & $\checkmark$ & \\
\hline 19 & & & $\checkmark$ & & & & & $\checkmark$ \\
\hline 20 & & & & & & & NS & NS \\
\hline 21 & & & & $\checkmark$ & & & $\checkmark$ & $\checkmark$ \\
\hline 22 & & & $\checkmark$ & & & & & $\checkmark$ \\
\hline 23 & & & & $\checkmark$ & & & $\checkmark$ & \\
\hline 24 & & & & $\checkmark$ & & & $\checkmark$ & \\
\hline 26 & & & $\checkmark$ & & & & & $\checkmark$ \\
\hline 29 & & & & $\checkmark$ & $\checkmark$ & & $\checkmark$ & \\
\hline 30 & & & & $\checkmark$ & & & $\checkmark$ & \\
\hline 31 & & $\checkmark$ & & $\checkmark$ & & & $\checkmark$ & \\
\hline 32 & & & & & & $\checkmark$ & & $\checkmark$ \\
\hline 33 & & $\checkmark$ & $\checkmark$ & & & & & $\checkmark$ \\
\hline 34 & & $\checkmark$ & & & NS & NS & NS & NS \\
\hline 35 & & & & $\checkmark$ & NS & NS & NS & NS \\
\hline
\end{tabular}

$\checkmark$ means that the strain was detected in the faeces of the subject, NS means "no sample available" and A and B correspond to L. paracasei NCC2461 and B. lactis SD5219, respectively.

IgG4 was induced after treatment with any of the probiotics and no difference on this parameter was observed between the two probiotic treatments (data not shown).

\section{Discussion}

This study aimed at assessing the effect of two probiotic preparations on established allergic rhinitis in the frame of a well-reproducible and well-standardized nasal provocation test (NPT). The L. paracasei NCC2461 strain was selected on the basis of preclinical in vivo data. NCC2461 mono-associated germ-free mice developed a TH1-like serum immunoglobulin profile (increased L. paracasei-specific IgG2a level as compared to specific IgG1 level) [32]. A beneficial effect of orally administered $L$. paracasei strain NCC2461 was established in a mouse model of asthma [33] and more importantly, a first pilot proof of efficacy with $L$. paracasei strain NCC2461 was obtained in volunteers suffering from allergic rhinitis that showed significant improvement in nasal congestion and modulation of immune biomarkers [23]. The combination of $25 \% \mathrm{~L}$. acidophilus ATCC SD5221 and 75\% B. lactis ATCC SD5219 was tested in the same setting, based on its 

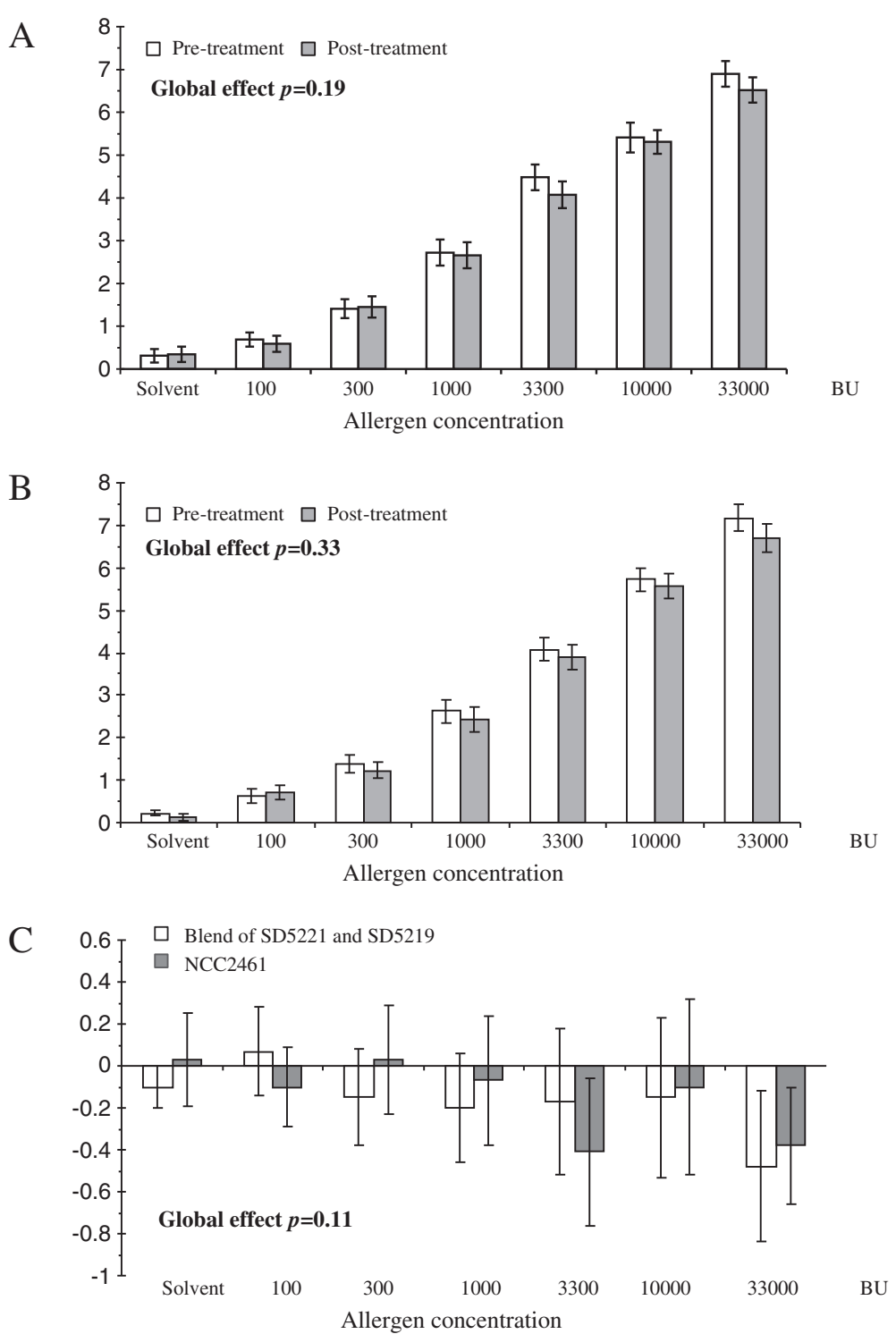

Figure 3 Mean VAS nasal congestion scores according to the allergen dose level (mean \pm SEM) pre- and post-treatment with $L$. paracasei NCC2461 (A), with the blend of L. acidophilus SD5221 and B. lactis SD5219 (B), and pre- minus post-treatment difference between the blend of $L$. acidophilus SD5221 and B. lactis SD5219 (open bars) and L. paracasei NCC2461 (black bars) treatments (ITT analysis) (C).

anti-inflammatory activity and TH1-type stimulating effect in a PBMC model (personal communication). Moreover a study recently conducted in children with birch pollen allergy showed that a 4 month-consumption of the probiotic mix, starting before the onset of the birch pollen season and continuing during the pollen season, tended to alleviate allergic rhinitis symptoms, and significantly prevented pollen-induced eosinophil infiltration in the nasal mucosa [24].

Although in the present study no significant effect was observed on the primary outcome i.e. nasal congestion, treatment with $L$. paracasei NCC2461 positively modulated several secondary endpoints: it significantly improved a key subjective clinical feature of allergic rhinitis, nasal pruritus, significantly decreased the percentage of leukocytes in nasal fluid, enhanced allergen specific IL-10 secretion by PBMC and tended to decrease IL- 5 in the nasal fluid, both biological effects supporting the improvement in nasal pruritus. An increase in IL-5, IL-13, IL-10 and IL- 8 by grass pollen stimulated PBMCs was interestingly observed after the L. paracasei NCC2461 treatment. Actually, it is well demonstrated that the induction of IL-10 is dependent on prior activation of $\mathrm{TH} 2$ cytokines [34]. These increases are thus not unexpected. The TH2 type of cytokine response induced by NCC2461 treatment on grass pollen stimulated $\mathrm{PBMC}$, further supported by the 


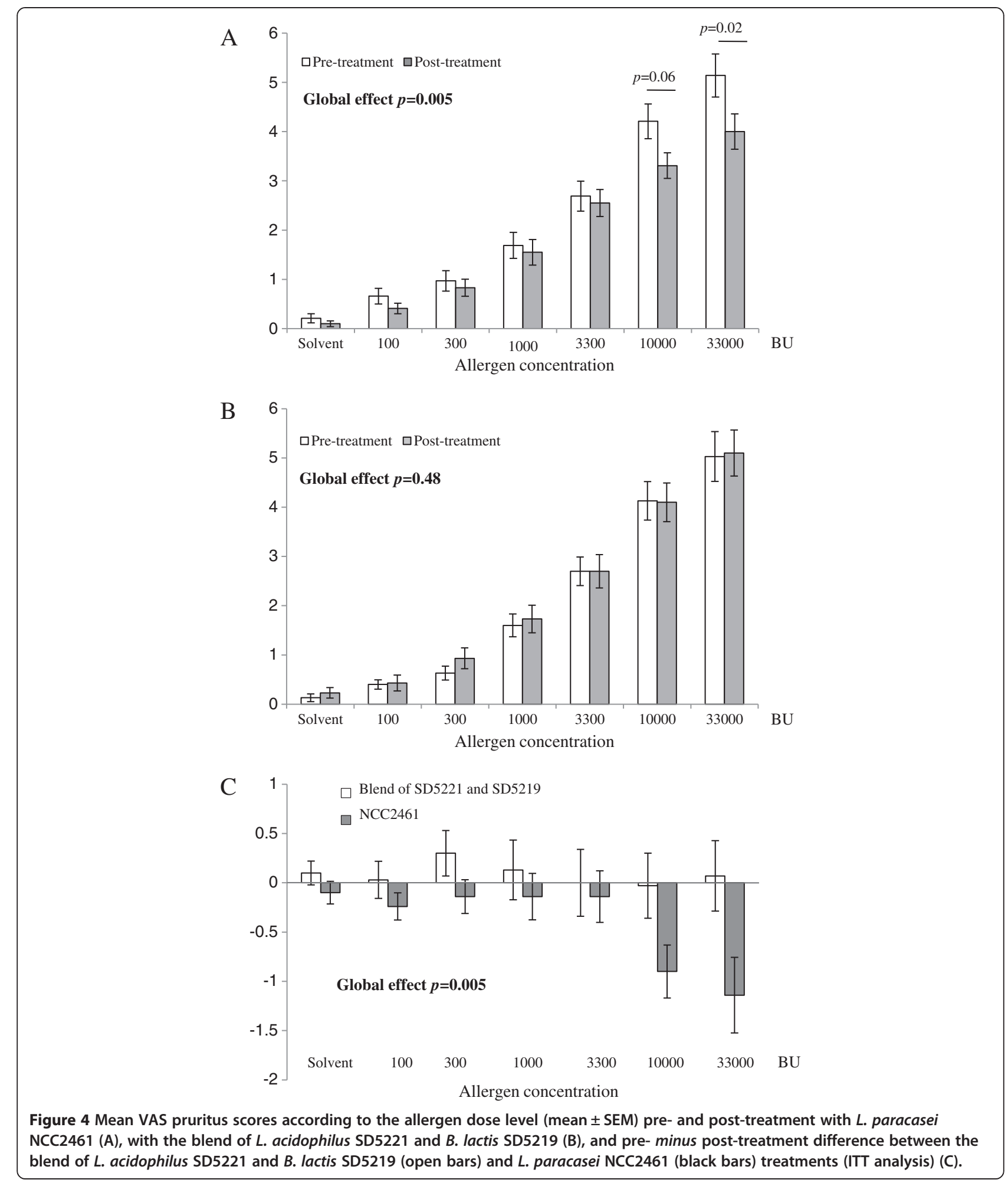

significant (but small and thus most likely biologically non relevant) increase in grass pollen specific IgE, may be surprising at first glance. Yet, this $\mathrm{TH} 2$ profile was not observed in the induction site, i.e. the nasal mucosa, and strictly concerned the systemic response. Interestingly,
Marschan et al. also described an elevation of plasma IgE and IL-10 in infants with a family history of allergy. Infants were given probiotics for 6 months after delivery from a mother having received the same probiotics for 1 month before delivery [35]. 


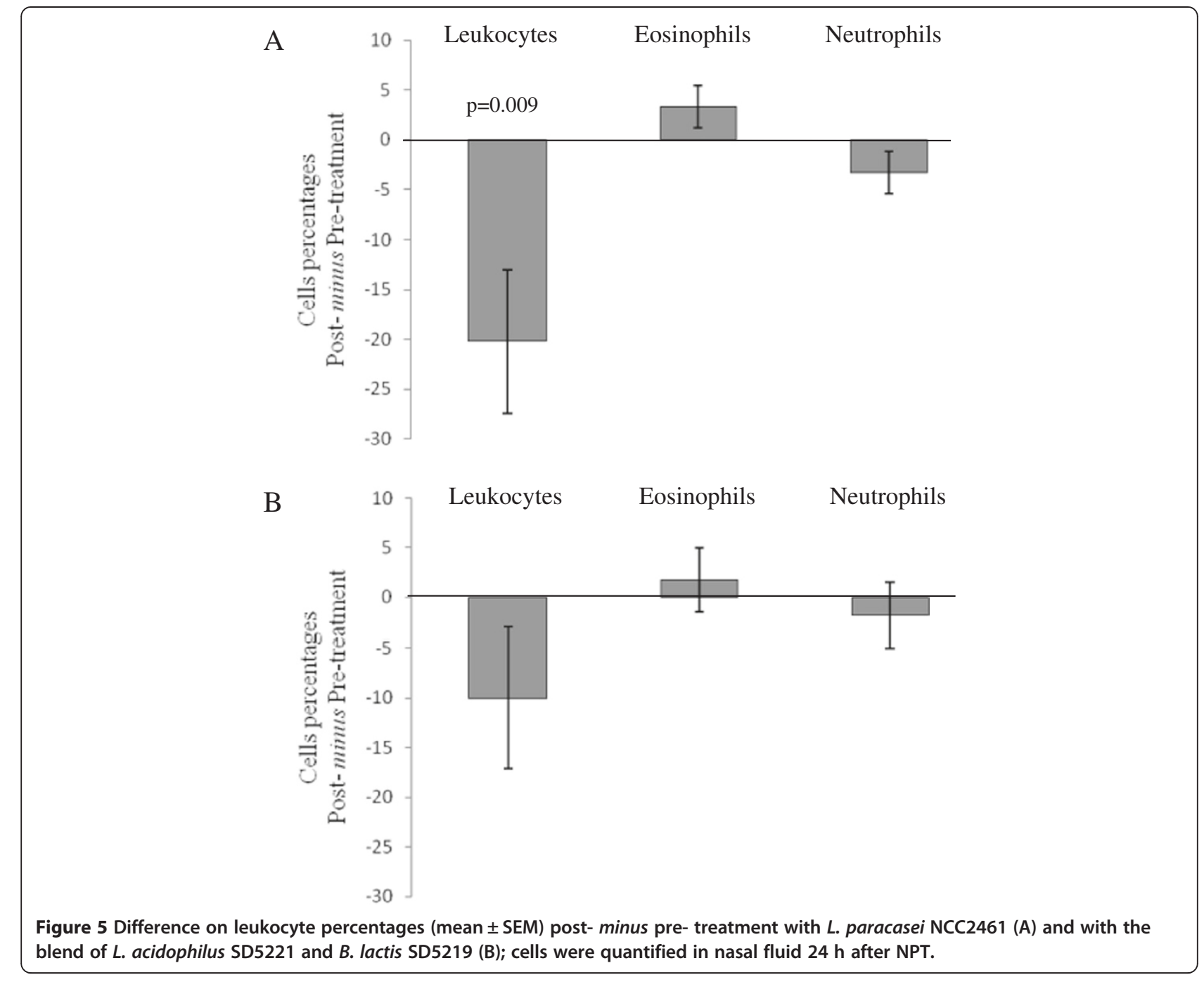

L. paracasei NCC2461 treatment was superior to the blend of L. acidophilus ATCC SD5221 and B. lactis ATCC SD5219 used as comparator for improvement of nasal pruritus, allergen specific IL-8 and IL-10 secretion by PBMC after stimulation with allergen and recall antigen, respectively. Of note, objective clinical parameters determined during NPT were not improved by this treatment, reflecting their lower sensitivity, as compared to the VAS evaluation.

As compared to other studies where probiotics were administered [36], the treatment period in the present clinical trial was of relatively short duration. We showed in a recent study [22] that the duration of the probiotic treatment may have an impact on the beneficial effect on both symptoms and immune markers. The beneficial results obtained on several parameters with $L$. paracasei NCC2461 after only 4 weeks of treatment can thus be considered as encouraging. Moreover, the level of improvement of nasal pruritus observed in this study after
L. paracasei NCC2461 consumption was comparable to the improvement observed after treatment with the anti$\mathrm{H} 1$ desloratadine [25] and is then likely to have a real clinical significance. We have also previously reported an effect of the $L$. paracasei NCC2461 strain in a crossover, placebo controlled trial where the effect was more pronounced on nasal congestion with the same duration of treatment. i.e. four weeks. Apart from the different study design, a possible reason for the different effects can be accounted to the complexity of allergic rhinitis as a disease and the fact that not every subject suffering from allergic rhinitis will manifest with the same predominant nasal allergy symptom.

Treatment with the blend of $L$. acidophilus ATCC SD5221 and B. lactis ATCC SD5219 was not able to induce comparable benefits, although it tended to reduce nasal symptoms in a different study setting; albeit only with a longer duration (4 months) of administration [24]. The discrepancy between the two studies with this 
Table 3 Difference in cytokine secretion by ex vivo restimulated PBMC isolated from subjects post- minus pre-consumption of either L. paracasei NCC2461 or a blend of L. acidophilus SD5221 and B. lactis SD5219

\begin{tabular}{|c|c|c|c|c|c|c|c|c|}
\hline \multicolumn{9}{|c|}{ L. paracasei NCC2461 treatment } \\
\hline & \multicolumn{2}{|c|}{ Allergen $\mathbf{n} \mathbf{n}$} & \multirow{2}{*}{$\begin{array}{l}\text { Median } \\
0.00\end{array}$} & \multirow{2}{*}{$\begin{array}{l}\text { Rob. SD } \\
0.77\end{array}$} & \multirow{2}{*}{$\begin{array}{l}\text { SE median } \\
0.18\end{array}$} & \multirow{2}{*}{$\begin{array}{l}\text { Min. } \\
-69.61\end{array}$} & \multirow{2}{*}{$\begin{array}{l}\text { Max. } \\
6.96\end{array}$} & \multirow{2}{*}{$\begin{array}{l}\boldsymbol{p} \text {-value } \\
0.92\end{array}$} \\
\hline \multirow[t]{3}{*}{ IL5 (pg/ml) } & NS & 29 & & & & & & \\
\hline & MM & 29 & 26.58 & 83.51 & 19.44 & -79.91 & 462.69 & 0.09 \\
\hline & GR & 29 & 85.51 & 150.53 & 35.03 & -144.70 & 529.87 & 0.005 \\
\hline \multirow[t]{3}{*}{ IL8 (pg/ml) } & NS & 29 & 3328 & 20686 & 4814 & -137069 & 51061 & 0.45 \\
\hline & MM & 29 & 51074 & 177356 & 41277 & -331659 & 489670 & 0.41 \\
\hline & $G R$ & 29 & 85702 & 156506 & 36424 & -370412 & 1328885 & 0.004 \\
\hline \multirow[t]{3}{*}{ IL10 (pg/ml) } & NS & 29 & 0.00 & 0.46 & 0.11 & -1.04 & 2.48 & 0.89 \\
\hline & MM & 29 & 1.80 & 4.80 & 1.12 & -5.93 & 16.14 & 0.01 \\
\hline & $G R$ & 29 & 3.03 & 6.63 & 1.54 & -11.60 & 25.20 & 0.008 \\
\hline \multirow[t]{3}{*}{ IL13 (pg/ml) } & NS & 29 & 0.00 & 4.52 & 1.05 & -87.11 & 116.31 & 0.97 \\
\hline & $\mathrm{MM}$ & 29 & 49.93 & 288.58 & 67.16 & -1000.90 & 1080.11 & 0.09 \\
\hline & $G R$ & 29 & 266.00 & 332.54 & 77.39 & -466.57 & 908.99 & 0.001 \\
\hline \multirow[t]{3}{*}{ IFN- $\gamma(\mathrm{pg} / \mathrm{ml})$} & NS & 29 & 0.00 & 0.00 & 0.00 & -106.58 & 169.48 & 0.86 \\
\hline & MM & 29 & 1644.34 & 8456.16 & 1868.04 & -30350.99 & 43924.50 & 0.15 \\
\hline & $G R$ & 29 & -165.66 & 1495.68 & 348.10 & -6365.44 & 10243.64 & 0.36 \\
\hline \multicolumn{9}{|c|}{ Blend of L. acidophilus SD5221 and B. lactis SD5219 treatment } \\
\hline & Aller & & Median & Rob. SD & SE median & Min. & Max. & $p$-value \\
\hline \multirow[t]{3}{*}{ IL5 (pg/ml) } & NS & 31 & 0.00 & 0.83 & 0.19 & -5.51 & 17.17 & 0.29 \\
\hline & MM & 31 & 0.23 & 77.11 & 17.36 & -378.76 & 221.79 & 0.98 \\
\hline & GR & 31 & 29.36 & 170.38 & 38.35 & -674.14 & 1686.96 & 0.20 \\
\hline \multirow[t]{3}{*}{ IL8 (pg/ml) } & NS & 31 & 2353 & 22598 & 5087 & -240155 & 73699 & 0.84 \\
\hline & $\mathrm{MM}$ & 31 & -26620 & 166240 & 37421 & -353181 & 1134834 & 0.85 \\
\hline & $\mathrm{GR}$ & 31 & -7132 & 153404 & 34532 & -1326885 & 300638 & 0.96 \\
\hline \multirow[t]{3}{*}{ IL10 (pg/ml) } & NS & 31 & 0.00 & 0.41 & 0.09 & -11.18 & 3.57 & 0.42 \\
\hline & MM & 31 & 0.84 & 6.02 & 1.36 & -13.71 & 11.87 & 0.58 \\
\hline & $G R$ & 31 & 0.60 & 5.95 & 1.34 & -14.84 & 17.89 & 0.75 \\
\hline \multirow[t]{3}{*}{ IL13 (pg/ml) } & NS & 31 & 0.00 & 0.794 & 0.18 & -10.672 & 70.676 & 0.04 \\
\hline & MM & 31 & 59.10 & 322.66 & 72.63 & -1023.04 & 4155.62 & 0.42 \\
\hline & $G R$ & 31 & 151.92 & 374.60 & 84 & -721.54 & 924.79 & 0.14 \\
\hline \multirow[t]{3}{*}{ IFN- $\nu \quad(p g / m l)$} & NS & 31 & 0.00 & 0.46 & 0.10 & -60.03 & 252.65 & 0.41 \\
\hline & MM & 31 & -365.81 & 9372.17 & 2109.69 & -45256.46 & 21604.04 & 0.59 \\
\hline & GR & 31 & -200.91 & 1261.78 & 284.03 & -8287.66 & 3864.57 & 0.59 \\
\hline
\end{tabular}

Samples were collected 24 hours after NPT (post- minus pre-treatment differences; ITT data set). NS: non stimulated PBMCs; MM: PBMCs stimulated with vaccine antigens cocktail; GR: PBMCs stimulated with grass pollen (ITT analysis).

probiotic mix can possibly be explained by age of the subjects, as well as timing and duration of probiotic supplementation and possibly with the challenging clinical trial setting of nasal provocation testing.

Due to the clinical trial setting used (comparison of two probiotics preparations), both investigators and subjects (although blinded with regard to the probiotic preparation) were aware that the aim of the study was to test probiotics, and thus a possible placebo effect cannot be excluded. However, this possible placebo effect would have been similar in the two groups and thus did most likely not impact on the difference in terms of beneficial effect observed between the 2 groups.

These results strongly suggest an intrinsic effect of L. paracasei NCC2461 treatment via yet undefined mechanisms. We may postulate a role for specific TLR engagement by uncharacterized TLR ligands from the $L$. paracasei NCC2461 strain, leading to an effect that was not apparent after treatment with the blend of $L$. acidophilus ATCC SD5221 and B. lactis ATCC SD5219 
[37]. To date, the involvement of TLR by probiotics mainly concerns TLR2 and TLR4, responsible for the generation of TH1 cytokines by PBMCs [38]. We cannot exclude that the secretion of IL-10 might play a marked anti-inflammatory role in vivo, thus contributing to limit the expansion of the systemic TH2 subset [39], that in turn would reduce local responses, i.e. nasal pruritus and nasal mucosa inflammation. The increase in cytokine secretion by PBMC or the enhanced serum IgE was very limited and of doubtful clinical significance, and furthermore expectable in patients with established allergen specific TH2 T cell response. Moreover, variation in cytokine production was not significantly different when both treatments were compared, except for IL-8 production. The role of inducible regulatory $\mathrm{T}$ cells [40] or of TH17 cells [41] has not been evaluated in this study but is strongly suggested by the enhanced production in IL-10 post NCC2461 treatment and would certainly deserve attention in the future.

In conclusion, the results presented here reinforced the promising data already obtained in a preliminary study with $L$. paracasei NCC2461 consumption [23]. Altogether, L. paracasei NCC2461 consumption was well tolerated and showed a therapeutic potential. We have also evaluated the clinical trial setting of nasal provocation tests as a possible way to examine candidate probiotic strains and feel that this methodology, routinely employed for demonstrating efficacy of mainstream pharmaceutical treatment, could offer the probiotic field flexibility in terms of conducting efficacy clinical trials as the window period of seasonal allergens (grass, birch pollen) is sometimes rather short to conduct field trials. However, we foresee a combined approach of nasal provocation testing and field trials to validate the scientific findings observed in either setting. Future seasonal clinical trials are then warranted to confirm the beneficial effect of $L$. paracasei NCC2461 consumption, including assessment of quality of life, combined symptom and medications scores and key immune biomarkers.

\section{Abbreviations}

NPT: Nasal provocation test; SPT: Skin prick test; VAS: Visual analog scale; PNIF: Peak nasal inspiratory flow; MCA: Mean cross-sectional area.

\section{Competing interests}

The authors declare that they have no competing interests.

\section{Authors' contributions}

All authors have been involved in drafting the manuscript and revising it critically; all authors have given approval of the submitted version of the paper. Furthermore, YP and JW carried out the clinical assessments, SN, AM, RA and FS contributed to acquisition of funding, participated to conception and design of the study and have been involved in the interpretation of data. RA, NB and VA established key laboratory techniques, carried out immune analyses and revised illustrations. BB and RB were involved in bacterial strains detection. JM performed the statistical analysis.

\section{Acknowledgments}

We thank A. C. Ouwehand and D. Carcano for fruitful discussions and Danisco Cultures (Madison, USA) for kindly providing the probiotic blend of L. acidophilus and B. lactis. We appreciated critical reading of the manuscript by A. C. Ouwehand and statistical analyses by JM. Enslens. We acknowledge the skillful contribution of G. Reuteler and I. Rochat for the microbial analyses.

\section{Author details}

'Division of Immunology and Allergy, Centre Hospitalier Universitaire Vaudois, Lausanne, Switzerland. ${ }^{2}$ Nestlé Research Centre, Lausanne, Switzerland. ${ }^{3}$ Current address: AgResearch Ltd, Food and Bio-based Products, Hamilton, New Zealand.

Received: 5 June 2013 Accepted: 17 December 2013

Published: 6 January 2014

\section{References}

1. Kalliomaki M, Antoine JM, Herz U, Rijkers GT, Wells JM, Mercenier A: Guidance for substantiating the evidence for beneficial effects of probiotics: prevention and management of allergic diseases by probiotics. J Nutr 2010, 140:713-721.

2. Laitinen K, Isolauri E: Management of food allergy: vitamins, fatty acids or probiotics? Eur J Gastroenterol Hepatol 2005, 17:1305-1311.

3. O'Flaherty JT: Lipid mediators of inflammation and allergy. Lab Invest 1982, 47:314-329.

4. Joint FAONHO Working Group Report on Drafting Guidelines for the Evaluation of Probiotics in Food. London, Canada; 2002.

5. Rautava S, Ruuskanen O, Ouwehand A, Salminen S, Isolauri E: The hygiene hypothesis of atopic disease-an extended version. J Pediatr Gastroenterol Nutr 2004, 38:378-388.

6. Bjorksten B, Sepp E, Julge K, Voor T, Mikelsaar M: Allergy development and the intestinal microflora during the first year of life. J Allergy Clin Immunol 2001, 108:516-520.

7. Kalliomaki M, Kirjavainen P, Eerola E, Kero P, Salminen S, Isolauri E: Distinct patterns of neonatal gut microflora in infants in whom atopy was and was not developing. J Allergy Clin Immunol 2001, 107:129-134.

8. Bisgaard H, Li N, Bonnelykke K, Chawes BL, Skov T, Paludan-Müller G, Stokholm J, Smith B, Krogfelt KA: Reduced diversity of the intestinal microbiota during infancy is associated with increased risk of allergic disease at school age. J Allergy Clin Immunol 2011, 128:646-652.

9. Abrahamsson TR, Jakobsson HE, Andersson AF, Björkstén B, Engstrand L, Jenmalm MC: Low diversity of the gut microbiota in infants with atopic eczema. J Allergy Clin Immunol 2012, 129:434-440.

10. Kalliomaki M, Salminen S, Arvilommi H, Kero P, Koskinen P, Isolauri E: Probiotics in primary prevention of atopic disease: a randomised placebo-controlled trial. Lancet 2001, 357:1076-1079.

11. Viljanen M, Savilahti E, Haahtela T, Juntunen-Backman K, Korpela R, Poussa T, Tuure T, Kuitunen M: Probiotics in the treatment of atopic eczema/dermatitis syndrome in infants: a double-blind placebo-controlled trial. Allergy 2005, 60:494-500.

12. Weston S, Halbert A, Richmond P, Prescott SL: Effects of probiotics on atopic dermatitis: a randomised controlled trial. Arch Dis Child 2005, 90:892-897.

13. Taylor AL, Dunstan JA, Prescott SL: Probiotic supplementation for the first 6 months of life fails to reduce the risk of atopic dermatitis and increases the risk of allergen sensitization in high-risk children: a randomized controlled trial. J Allergy Clin Immunol 2007, 119:184-191.

14. Michail SK, Stolfi A, Johnson T, Onady GM: Efficacy of probiotics in the treatment of pediatric atopic dermatitis: a meta-analysis of randomized controlled trials. Ann Allergy Asthma Immunol 2008, 101:508-516.

15. Ismail $I H$, Licciardi PV, Tang ML: Probiotic effects in allergic disease. J Paediatr Child Health 2013, 49:709-715.

16. Helin T, Haahtela S, Haahtela T: No effect of oral treatment with an intestinal bacterial strain, Lactobacillus rhamnosus (ATCC 53103), on birch-pollen allergy: a placebo-controlled double-blind study. Allergy 2002, 57:243-246.

17. Tamura M, Shikina T, Morihana T, Hayama M, Kajimoto O, Sakamoto A, Kajimoto Y, Watanabe O, Nonaka C, Shida K, Nanno M: Effects of probiotics on allergic rhinitis induced by Japanese cedar pollen: randomized double-blind, placebo-controlled clinical trial. Int Arch Allergy Immunol 2007, 143:75-82. 
18. Wang MF, Lin HC, Wang YY, Hsu CH: Treatment of perennial allergic rhinitis with lactic acid bacteria. Pediatr Allergy Immunol 2004, 15:152-158.

19. Peng GC, Hsu CH: The efficacy and safety of heat-killed Lactobacillus paracasei for treatment of perennial allergic rhinitis induced by housedust mite. Pediatr Allergy Immunol 2005, 16:433-438.

20. Xiao JZ, Kondo S, Yanagisawa N, Takahashi N, Odamaki T, Iwabuchi N, Miyaji K, Iwatsuki K, Togashi H, Enomoto K, Enomoto T: Probiotics in the treatment of Japanese cedar pollinosis: a double-blind placebo-controlled trial. Clin Exp Allergy 2006, 36:1425-1435.

21. Ivory K, Chambers SJ, Pin C, Prieto E, Arques JL, Nicoletti C: Oral delivery of Lactobacillus casei Shirota modifies allergen-induced immune responses in allergic rhinitis. Clin Exp Allergy 2008, 38:1282-1289.

22. Singh A, Hacini-Rachinel F, Gosoniu ML, Bourdeau T, Holvoet S, Doucet-Ladeveze R, Beaumont M, Mercenier A, Nutten S: Immune-modulatory effect of probiotic Bifidobacterium lactis NCC2818 in individuals suffering from seasonal allergic rhinitis to grass pollen: an exploratory, randomized, placebo-controlled clinical trial. Eur J Clin Nutr 2013, 67:161-167.

23. Wassenberg J, Nutten S, Audran R, Barbier N, Aubert V, Moulin J, Mercenier A, Spertini F: Effect of Lactobacillus paracasei ST11 on a nasal provocation test with grass pollen in allergic rhinitis. Clin Exp Allergy 2011, 41:565-573.

24. Ouwehand AC, Nermes M, Collado MC, Rautonen N, Salminen S, Isolauri E: Specific probiotics alleviate allergic rhinitis during the birch pollen season. World J Gastroenterol 2009, 15:3261-3268.

25. Deruaz C, Leimgruber A, Berney M, Pradervand E, Spertini F: Levocetirizine better protects than desloratadine in a nasal provocation with allergen. J Allergy Clin Immunol 2004, 113:669-676.

26. Zweiman B, Getsy J, Kalenian M, Lane A, Schwartz LB, Doty R, Lanza D: Nasal airway changes assessed by acoustic rhinometry and mediator release during immediate and late reactions to allergen challenge. J Allergy Clin Immunol 1997, 100:624-631.

27. Ganslmayer M, Spertini F, Rahm F, Terrien MH, Mosimann B, Leimgruber A: Evaluation of acoustic rhinometry in a nasal provocation test with allergen. Allergy 1999, 54:974-979.

28. Terrien MH, Rahm F, Fellrath JM, Spertini F: Comparison of the effects of terfenadine with fexofenadine on nasal provocation tests with allergen. J Allergy Clin Immunol 1999, 103:1025-1030.

29. Erin EM, Zacharasiewicz AS, Nicholson GC, Tan AJ, Higgins LA, Williams TJ, Murdoch RD, Durham SR, Barnes PJ, Hansel TT: Topical corticosteroid inhibits interleukin-4, -5 and -13 in nasal secretions following allergen challenge. Clin Exp Allergy 2005, 35:1608-1614.

30. Bensch GW, Nelson HS, Borish LC: Evaluation of cytokines in nasal secretions after nasal antigen challenge: lack of influence of antihistamines. Ann Allergy Asthma Immunol 2002, 88:457-462.

31. Simpson PJ, Fitzgerald GF, Stanton C, Ross RP: The evaluation of a mupirocin-based selective medium for the enumeration of bifidobacteria from probiotic animal feed. J Microbiol Methods 2004, 57:9-16.

32. Ibnou-Zekri N, Schiffrin EJ, Von der Weid T: Divergent patterns of colonization and immune response elicited from two intestinal Lactobacillus strains that display similar properties in vitro. Infect Immun 2003, 71:428-436.

33. Pellaton C, Nutten S, Thierry AC, Boudousquié C, Barbier N, Blanchard C, Corthésy B, Mercenier A, Spertini F: Intragastric and intranasal administration of lactobacillus paracasei NCC2461 modulates allergic airway inflammation in mice. Int J Inflam 2012, 2012:686739.

34. Aalberse RC, Stapel SO, Schuurman J, Rispens T: Immunoglobulin G4: an odd antibody. Clin Exp Allergy 2009, 39:469-477.

35. Marschan E, Kuitunen M, Kukkonen K, Poussa T, Sarnesto A, Haahtela T, Korpela R, Savilahti E, Vaarala O: Probiotics in infancy induce protective immune profiles that are characteristic for chronic low-grade inflammation. Clin Exp Allergy 2008, 38:611-618.

36. Vliagoftis $H$, Kouranos VD, Betsi Gl, Falagas ME: Probiotics for the treatment of allergic rhinitis and asthma: systematic review of randomized controlled trials. Ann Allergy Asthma Immunol 2008, 101:570-579.

37. Miettinen M, Veckman V, Latvala S, Sareneva T, Matikainen S, Julkunen I: Live Lactobacillus rhamnosus and Streptococcus pyogenes differentially regulate Toll-like receptor (TLR) gene expression in human primary macrophages. J Leukoc Biol 2008, 84:1092-1100.

38. Miettinen M, Matikainen S, Vuopio-Varkila J, Pirhonen J, Varkila K, Kurimoto M, Julkunen I: Lactobacilli and streptococci induce interleukin-12 (IL-12), IL-18, and gamma interferon production in human peripheral blood mononuclear cells. Infect Immun 1998, 66:6058-6062.
39. Amati L, Pepe M, Passeri ME, Mastronardi ML, Jirillo E, Covelli V: Toll-like receptor signaling mechanisms involved in dendritic cell activation: potential therapeutic control of T cell polarization. Curr Pharm Des 2006, 12:4247-4254

40. Ozdemir C, Akdis M, Akdis CA: T-cell response to allergens. Chem Immunol Allergy 2010, 95:22-44

41. Schmidt-Weber CB, Akdis M, Akdis CA: TH17 cells in the big picture of immunology. J Allergy Clin Immunol 2007, 120:247-254.

doi:10.1186/2045-7022-4-1

Cite this article as: Perrin et al.: Comparison of two oral probiotic preparations in a randomized crossover trial highlights a potentially beneficial effect of Lactobacillus paracasei NCC2461 in patients with allergic rhinitis. Clinical and Translational Allergy 2014 4:1.

\section{Submit your next manuscript to BioMed Central and take full advantage of:}

- Convenient online submission

- Thorough peer review

- No space constraints or color figure charges

- Immediate publication on acceptance

- Inclusion in PubMed, CAS, Scopus and Google Scholar

- Research which is freely available for redistribution

Submit your manuscript at www.biomedcentral.com/submit
C BioMed Central 